Neurosci Lett. 2013 May 24; 543: 172-176. doi:10.1016/j.neulet.2013.03.028.

\title{
Altered Cortical Communication in Amyotrophic Lateral Sclerosis
}

\author{
Stefanie Blain-Moraes ${ }^{a}$, George A. Mashoura, Heonsoo Lee ${ }^{a, c}$, Jane E. Huggins ${ }^{b}$, and \\ UnCheol Lee ${ }^{a}$ \\ aDepartment of Anesthesiology, University of Michigan Medical School, 7433 Med Sci I, 1150 \\ West Medical Center Drive, Ann Arbor, Michigan, 48105, United States of America \\ bepartment of Physical Medicine and Rehabilitation, University of Michigan, 1500 East Medical \\ Center Drive, Ann Arbor, Michigan, 48109, United States of America \\ 'Department of Physics, Pohang University of Science and Technology, Pohang, Gyeongbuk, \\ Korea
}

\begin{abstract}
Amyotrophic lateral sclerosis (ALS) is a disorder associated primarily with the degeneration of the motor system. More recently, functional connectivity studies have demonstrated potentially adaptive changes in ALS brain organization, but disease-related changes in cortical communication remain unknown. We recruited individuals with ALS and age-matched controls to operate a brain-computer interface while electroencephalography was recorded over three sessions. Using normalized symbolic transfer entropy, we measured directed functional connectivity from frontal to parietal (feedback connectivity) and parietal to frontal (feedforward connectivity) regions. Feedback connectivity was not significantly different between groups, but feedforward connectivity was significantly higher in individuals with ALS. This result was consistent across a broad electroencephalographic spectrum $(4-35 \mathrm{~Hz})$, and in theta, alpha and beta frequency bands. Feedback connectivity has been associated with conscious state and was found to be independent of ALS symptom severity in this study, which may have significant implications for the detection of consciousness in individuals with advanced ALS. We suggest that increases in feedforward connectivity represent a compensatory response to the ALS-related loss of input such that sensory stimuli have sufficient strength to cross the threshold necessary for conscious processing in the global neuronal workspace.
\end{abstract}

\section{Keywords}

Amyotrophic lateral sclerosis; directed functional connectivity; symbolic transfer entropy; frontoparietal connectivity; recurrent processing; electroencephalography

\footnotetext{
(C) 2013 Elsevier Ireland Ltd. All rights reserved.

Corresponding author: UnCheol Lee, Department of Anesthesiology, University of Michigan Medical School, 7433 Med Sci I, 1150 West Medical Center Drive, Ann Arbor, MI, 48105, uclee@med.umich.edu, Telephone: 1-734-764-8015.

Publisher's Disclaimer: This is a PDF file of an unedited manuscript that has been accepted for publication. As a service to our customers we are providing this early version of the manuscript. The manuscript will undergo copyediting, typesetting, and review of the resulting proof before it is published in its final citable form. Please note that during the production process errors may be discovered which could affect the content, and all legal disclaimers that apply to the journal pertain.
} 


\section{Introduction}

Amyotrophic lateral sclerosis (ALS) is an adult-onset, progressive disorder that is characterized by the degeneration of upper and lower motor neurons as well as the corticospinal tract [25]. ALS has traditionally been associated almost exclusively with neurodegeneration of the motor system. More recently, growing evidence of changes in executive function, behavior, language and other higher cognitive domains suggests that ALS is a multisystem disease that also affects cortical processing [1,27]. This concept has been supported by diffusion tensor imaging (DTI) and magnetic resonance imaging (MRI) studies that have demonstrated a widespread pattern of structural alterations in the corpus callosum, frontal, temporal and parietal lobes of patients with ALS $[2,3,8-10,14,16,19,31,32,40]$.

The functional correlates of these structural changes have been less extensively studied, and the effect of ALS on extramotor brain networks is largely unknown [38]. To date, neurophysiological studies of ALS have demonstrated patterns of functional adaptation and cortical plasticity such as abnormal recruitment and functioning of primary and nonprimary motor areas [20-22,33,39].

To gain further insight into how ALS alters exchange of information between two brain regions, we used symbolic transfer entropy to measure the directed functional brain connectivity between the frontal and parietal regions of individuals with ALS. Patterns of frontoparietal information exchange have been associated with states of consciousness $[7,23,41]$, and the ability to explicitly process external stimuli $[6,12,30]$. Alterations in these patterns may reflect either an underlying source of the changes in executive function, behavior and language in individuals with ALS, or a compensatory mechanism to maintain such cognitive processes $[1,27]$. Studies examining functional cortical changes in ALS have also been limited to the resting state or the performance of a motor task, which may not be representative of the changes associated with cognitive processing. In this study, participants performed the cognitive task of operating a brain-computer interface [15], a task that also reflects the priorities of communication and the maintenance of relationships in individuals living with ALS [5] and thus may be useful in developing a clinically-relevant model of cortical changes in ALS for translational research [24]. The goal of the present study was to test the hypothesis that ALS is associated with alterations in frontoparietal communication that can be assessed with electroencephalography during a cognitive task.

\section{Methods}

\subsection{Participants}

This study was approved by the University of Michigan Medical School Institutional Review Board. Written consent was obtained from all participants who had sufficient motor ability to provide it; those who did not provided oral consent. Two groups of individuals participated in this study: the first consisted of individuals with a clinical diagnosis of ALS, recruited from the University of Michigan Motor Neuron Disease Clinic; the second consisted of individuals without ALS, who were age-matched to the ALS participants. Neither group underwent neuropsychological testing as part of this study.

\subsection{Cognitive task}

All participants engaged in the cognitive task of spelling a 23-character sentence using a P300-based brain-computer interface (BCI) [15]. Participants were seated approximately 1 $\mathrm{m}$ from a computer monitor that displayed a $6 \times 6$ grid of letters and commands. Rows and columns of the grid flashed in a seemingly random manner, while participants counted the flashes of the desired letter or command. After a specified number of flashes, the BCI 
selected a letter by classifying the participant's EEG patterns and presented it on the screen. Participants were asked to correct any errors that were made during the task, until the sentence was completed correctly or until the maximum time of 10 minutes had elapsed. The task was repeated over three sessions occurring on three different days, with a new sentence being presented to the participant each time.

\subsection{Data acquisition and processing}

EEG was collected from a 16-channel electrode cap embedded with tin electrodes (ElectroCap International, Inc., Eaton, OH). All channels were referenced to the right mastoid and grounded to the left mastoid. Impedance for each channel was reduced below $10.0 \mathrm{k} \Omega$ before testing began. Signals were collected using a g.tec (Guger Technologies, Graz, Austria) 16channel biosignal amplifier at a rate of $256 \mathrm{~Hz}$, and bandpass filtered offline between $4 \mathrm{~Hz}$ and $35 \mathrm{~Hz}$. Independent component analysis (ICA) was used to filter EOG artifact from all EEG channels. Subsequently, only electrodes from the frontal and parietal regions (F3, Fz, $\mathrm{F} 4, \mathrm{P} 3, \mathrm{Pz}, \mathrm{P} 4, \mathrm{PO} 7$ and PO8) were used for analysis.

\subsection{EEG data analysis}

Directed functional connectivity analysis-To assess alterations in cortical connectivity in individuals with ALS, we measured directed functional connectivity between the frontal and parietal regions of the brain. We quantified the degree of dependence of frontal regions on parietal regions and vice-versa using normalized symbolic transfer entropy (nSTE), a nonlinear and model-free metric based on information theory [34,36]. Briefly, using nSTE, signal $S_{1}$ is said to be causally related to signal $S_{2}$ if the inclusion of $S_{1}$ results in a better prediction of the future of $S_{2}$ than signal $S_{2}$ alone.

Let $X(t)$ and $Y(t), 0 \leq t \leq T$ represent the average EEG signal over the source and target regions of the brain, where $T$ is the duration of the signal. At time $t_{0}$, let $X^{P}$ and $Y^{P}$ represent the EEG signal when $0 \leq t \leq t_{0}$ - in other words, the signal past - and let $X^{F}$ and $Y^{F}$ represent the EEG signal when $t_{0} \leq t \leq T$ - in other words, the signal future. Transfer entropy from $X$ to $Y\left(T E_{X \rightarrow Y}\right)$, defined in equation (1), is the amount of mutual information between $X^{P}$ and $Y^{F}$ when $Y^{P}$ is known:

$$
T E_{X \rightarrow Y}=I\left(Y^{F} ; X^{P} \mid Y^{P}\right)=H\left(Y^{F} \mid Y^{P}\right)-H\left(Y^{F} \mid X^{P}, Y^{P}\right)
$$

where $H(X)$ is the entropy of signal $X$. In other words, $T E_{X \rightarrow Y}$ represents the amount of information added by the past of source signal $X$ when modeling the information between the past and future of target signal $Y$.

Symbolic transfer entropy (STE) is a more robust variation of transfer entropy, which avoids the need to subjectively select bin size in the probability calculation of mutual information [36,37]. In STE, each element in vectors $X^{P}, Y^{P}, X^{F}$ and $Y^{F}$ is replaced by the integer value of its relative rank in the vector. For example, $Y(t)$ is transformed to $Y(t)$ by replacing each element with its rank in ascending order $y_{j} \in[1,2, \cdots, T]$ for $j=1,2, \cdots T . Y(t)$ is then used in equation (2) to calculate $S T E_{X \rightarrow Y}$.

Bias removal was accomplished by removing from $S T E_{X \rightarrow Y}$ an estimate of the STE if no causal relationship existed between source and target signals [18]. Practically, this was accomplished by reversing the first and second half of $X^{P}$, resulting in $X_{\text {Shuff }}^{P}$ which retained the same signal characteristics as $X^{P}$ but was completely disconnected from $X^{F}$ and $Y^{F}$. STE with the shuffled source signal was calculated as in equation (2): 


$$
S T E_{X \rightarrow Y}^{\text {Shuff }}=H\left(Y^{F} \mid Y^{P}\right)-H\left(Y^{F} \mid X_{\text {Shuff }}^{P}, Y^{P}\right)
$$

Subtracting $S T E_{X \rightarrow Y}^{\text {Shuff }}$ from $S T E_{X \rightarrow Y}$ resulted in an unbiased estimate of STE. Finally, we normalize the unbiased STE by dividing it by the entropy within the target signal, resulting in $n S T E_{X \rightarrow Y}$.

$$
n S T E_{X \rightarrow Y}=\frac{S T E_{X \rightarrow Y}-S T E_{X \rightarrow Y}^{S h u f f}}{H\left(Y^{F} \mid Y^{P}\right)} \in[0,1]
$$

Intuitively, $n S T E_{X \rightarrow Y}$ represents the fraction of information in $Y^{F}$ not explained by its own past $\left(Y^{P}\right)$ and explained by the past of the source signal $X^{P}$.

If $X$ represents a parietal EEG signal and $Y$ represents a frontal EEG signal, we can calculate three values: 1) $n S T E_{X \rightarrow Y}$, herein referred to as feedforward connectivity; 2) $n S T E_{X \rightarrow Y}$, herein referred to as feedback connectivity; and 3) asymmetry, $A$, as defined in equation (4):

$$
A=\frac{n S T E_{Y \rightarrow X}-n S T E_{X \rightarrow Y}}{n S T E_{Y \rightarrow X}+n S T E_{X \rightarrow Y}}
$$

If $A$ is positive, feedback connectivity is dominant; if it is negative, feedforward connectivity is dominant. We evaluated average frontoparietal feedback and feedforward connectivity by calculating $\overline{n S T E}_{X \rightarrow Y}$ and $\overline{n S T E}_{Y \rightarrow X}$ for each participant:

$\overline{n S T E}_{Y \rightarrow X}=\frac{1}{n_{f} \cdot n_{p}} \sum_{(i, j)=1}^{n_{f}, n_{p}} n S T E_{i \rightarrow j}$, where $n_{f}=3$ and $n_{p}=5$.

For each participant, EEG collected from each of the three sessions was subdivided into four EEG datasets according to frequency: 1) total $(4 \mathrm{~Hz}-35 \mathrm{~Hz}) ; 2) \theta(4 \mathrm{~Hz}-8 \mathrm{~Hz}) ; 3) a(8 \mathrm{~Hz}$ $-13 \mathrm{~Hz})$; 4) $\beta(13 \mathrm{~Hz}-30 \mathrm{~Hz})$, yielding a total of 12 datasets per participant. $\delta$ frequencies were not included to minimize eye-blink artifact. $\overline{n S T E}_{X \rightarrow Y}, \overline{n S T E}_{X \rightarrow Y}$ and were $\bar{A}$ calculated for each dataset and averaged across sessions.

Determining appropriate embedding parameters-To calculate $n S T E$, three embedding parameters are required: embedding dimension $\left(d_{E}\right)$, time delay $(\tau)$ and prediction time $(\delta)$. In this study, we selected the parameter set that yielded maximum $n S T E_{Y \rightarrow X}$ by fixing the embedding dimension at $d_{E}=3$ and optimizing $\delta$ (from 1 to 100)

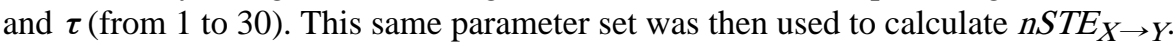

EEG spectral and coherence analysis-For each session, a Fast Fourier Transform (FFT) was performed and each frequency bin was normalized to total power. The resultant power spectral densities were subdivided into three frequency bands $(\theta$ : 4-8 Hz; a: 8-13 Hz; $\beta: 13-30 \mathrm{~Hz}$ ) for statistical analysis.

The total EEG spectrum and each frequency band were further analyzed by calculating the average magnitude-squared coherence $\left(\bar{C}_{X y}\right)$ between all frontal and parietal channels for each session, for each participant.

Statistical analysis-Feedforward connectivity, feedback connectivity and asymmetry results were analyzed using linear mixed models implemented in SAS (SAS Institute, Inc., Cary, NC, USA). The first model included diagnosis of ALS, participant age, task accuracy and session as fixed effects; participant as a random effect; and session as a repeated 
measures factor. The second model was limited to participants with ALS and included participant age, task accuracy, session and ALSFRS-R score as fixed effects; participant as a random effect; and session as a repeated measures factor.

Differences in the power spectral densities between individuals with ALS and age-matched controls were analyzed separately for each frequency band using a linear mixed model with diagnosis of ALS and session as fixed effects; participant as a random effect; and session as a repeated measures factor. Results were considered statistically significant at $\mathrm{p}<0.05$.

\section{Results}

Eleven individuals with a clinical diagnosis of ALS (7 male; $62+9$ years; ALSFRS-R score: $28+7)$ and 9 individuals without ALS (7 male; $61+10$ years) participated in this study. Participant characteristics and task performance information are shown in Table 1. There was no significant difference in accuracy of performance $(p=0.33)$ or self-reported concentration on the task $(p=0.39)$ between individuals with ALS and the controls.

\section{Directed functional connectivity patterns are altered between individuals with ALS and age-matched controls}

Feedforward connectivity, feedback connectivity and asymmetry were calculated using the total EEG spectrum for all 20 participants, over all three sessions. As shown in Figure 1a, feedback connectivity was not significantly different between individuals with ALS and agematched controls $(p=0.15)$; however, individuals with ALS demonstrated significantly greater feedforward connectivity $(p=0.004)$ and significantly lower asymmetry $(p=0.004)$ in comparison to the age-matched controls. This pattern of significantly altered cortical connectivity was consistently observed across $\theta, \alpha$ and $\beta$ frequency bands. Magnitudesquared coherence was not significantly different in the full EEG spectrum between individuals with $\operatorname{ALS}\left(\bar{C}_{x y}=0.58+0.15\right)$ and controls $\left(\bar{C}_{X y}=0.49+0.10\right)$, nor in any of the three frequency bands. Trial, age and task accuracy did not have a significant effect on feedforward connectivity, feedback connectivity or asymmetry. Importantly, the increase in feedforward connectivity was also independent of ALSFRS-R scores $(p=0.86)$.

\section{The difference in directed functional connectivity is not attributable to spectral differences in the electroencephalogram}

We assessed whether changes in directed functional connectivity between individuals with ALS and age-matched controls were associated with differences in the background EEG spectrum. No changes of the background EEG could be detected between individuals with ALS and age-matched controls (Figure 1b). These results suggest that the differences in cortical communication observed in individuals with ALS reflect a functional change in the underlying brain dynamics, rather than a change in the level of activation of the EEG.

\section{Discussion}

This is the first study to demonstrate that patterns of cortical communication are significantly altered in individuals with ALS during the performance of a cognitive task. These results are consistent with previous studies that have used whole brain analysis of functional connectivity to gain a better understanding of the disease mechanisms of ALS. These studies have demonstrated a variety of patterns of adaptation of the ALS brain: resting-state functional MRI (fMRI) studies have demonstrated both decreased and increased functional connectivity in default-mode and sensorimotor networks in ALS $[14,26]$. Others have shown that ALS results in increased functional connectivity within regions of decreased structural connectivity, spanning sensorimotor, premotor, prefrontal 
and thalamic regions [14], and decreased functional connectivity within the right frontoparietal network [38]. However, functional connectivity alone reveals statistical covariation of the activity in two cortical regions, which does not necessarily imply that there is information transfer or causal influence between them. Furthermore, changes in functional connectivity between two brain regions can be observed in the absence of a change in the underlying brain network (e.g. in the case of increase in signal-to-noise ratio, or increased amplitude of neuronal fluctuations), limiting the conclusions that can be drawn [17].

The current study advances the field by showing alterations of cortical communication, i.e. an exchange of information, within the functional organization of the ALS brain. Using nSTE with appropriate bias removal, we were able to discern patterns of directed influence between frontal and parietal brain regions, and demonstrated unchanged feedback communication but increased feedforward communication in individuals with ALS compared to controls. The increase in feedforward connectivity accounts for the decreased asymmetry (the ratio of feedback to feedforward connectivity) in individuals with ALS.

Previous research has suggested that feedforward communication represents incoming sensory data, while feedback communication plays a modulating role in the selection and contextual interpretation of information $[28,29,35]$. Impaired feedback communication has been observed in individuals with diminished states of consciousness, such as those under general anesthesia[23] and those in vegetative state [7]. The similar level of feedback communication between individuals with ALS and controls is an expected result, as the individuals with ALS who participated in this study did not experience impaired or heightened states of consciousness relative to healthy individuals. Our results demonstrate that the magnitude of overall feedback communication is independent of ALSFRS-R score, which may have significant implications for the detection of consciousness in individuals with such advanced ALS that no motor behavior is under their voluntary control.

This study does not address the underlying neural mechanisms that cause the observed augmentation in feedforward communication in individuals with ALS. It is not clear whether these changes are reflective of pathology, compensation or epiphenomenon. We interpret our results in light of the global neuronal workspace framework recently proposed by Dehaene and colleagues. This model posits the existence of workspace neurons that are distributed throughout the whole cortex, but with higher density in the parietal, frontal, and cingulate cortices [11,12]. Conscious processing of an external stimulus requires that two conditions are met: there must be neural activity within the sensory cortex of the respective modality and there must be entry into the global neuronal workspace, leading to long-range communication between the distributed workspace neurons. Without entry into and the coherent activity of this global workspace network, stimuli cannot be consciously processed by the subject $[4,13]$. It is hypothesized that entry into the global workspace is regulated in part by a threshold phenomenon, and activity above a specific threshold can enter the workspace in a bottom-up manner. Individuals with ALS likely have diminished levels of input into the global workspace as a result of the loss of motor-related phenomenon, such as feedback from movement and proprioception, and thus may be susceptible to diminished levels of conscious processing of external stimuli. In the present study, no significant difference was found in cognitive task performance accuracy between individuals with ALS and age-matched controls, suggesting that both groups had equivalent conscious processing of the visual stimuli associated with the task. We hypothesize that the observed increase in feedforward communication compensates for ALS-related loss of input into the global workspace such that sensory stimuli are represented with sufficient strength to cross the threshold necessary for conscious processing. 
An alternate explanation to the observed augmentation of feedforward communication may be drawn from the conclusions of a study by Douaud and colleagues demonstrating increases in functional connectivity in individuals with ALS [14]. Based upon studies that have revealed an abrupt increase in cortical hyperexcitability in the months prior to ALS symptom onset [42], and reduced attenuation to transcranial double magnetic stimulation in individuals with ALS [43], Douaud suggests that increases in functional connectivity may arise as a result of a loss of cortical inhibitory neural influence in ALS. It is possible that the increased feedforward communication observed in this study is similarly reflective of ALS pathogenesis, as opposed to a compensatory response. However, if this were indeed the underlying mechanism of increased feedforward communication, one would expect to see a corresponding increase in feedback communication due to the global and directionindependent nature of the inhibitory loss, which was not observed in this study. The preferential increase in parietal-to-frontal feedforward communication supports the hypotheses that changes in both functional connectivity and directed functional connectivity are reflective of adaptive and compensatory mechanisms in the ALS brain, as opposed to global pathogenesis.

The results of this study need to be interpreted in light of several important limitations. Data were collected from a small subject sample, using a low spatial resolution of 16 EEG channels which did not cover all frontal and parietal regions. EEG from posterior regions, which could yield important information about the effects of the visual stimuli associated with the BCI task, was not considered. Finally, while our analysis applied surrogate data tests, nSTE is still susceptible to the general limitations of quantitative EEG analysis (e.g. volume conduction, EEG reference).

In conclusion, this study used a measure of directed functional connectivity to investigate changes in cortical communication associated with ALS. These data provide the most detailed account to date of how information exchange between the frontal and parietal regions of the brain is altered as a result of this degenerative condition. The results demonstrate a significant preferential increase in feedforward communication, which may reflect compensatory mechanisms that the ALS brain uses to maintain unimpaired conscious processing of external stimuli.

\section{Acknowledgments}

This work was supported by Canadian Institutes of Health Research grant FRN: 123751 (SBM, GAM); the National Institute of Child Health and Human Development, the National Institutes of Health grant no. R21HD054697 (JEH); the Department of Education's National Institute on Disability and Rehabilitation Research grant no. H133G090005 (JEH); National Institute of General Medical Sciences, National Institutes of Health grant no. R01GM098578-01 (GAM, UL).

\section{References}

[1]. Abrahams S, Goldstein LH, Suckling J, Ng V, Simmons A, Chitnis X, et al. Frontotemporal white matter changes in amyotrophic lateral sclerosis. J. Neurol. 2005; 252:321-331. [PubMed: 15739047]

[2]. Agosta F, Pagani E, Petrolini M, Caputo D, Perini M, Prelle A, et al. Assessment of white matter tract damage in patients with amyotrophic lateral a diffusion tensor MR imaging tractography study. J Neuroradiol. 2010; 31:1457-1461.

[3]. Agosta F, Pagani E, Rocca MA, Caputo D, Perini M, Salvi F, et al. Voxel-based morphometry study of brain volumetry and diffusivity amyotrophic lateral sclerosis patients with mild disability. Brain Mapp. 2007; 28:1430-1438.

[4]. Baars, BJ. A Cognitive Theory of Consciousness. Cambridge University Press; 1993. 1st Paperback Edition, 1993 
[5]. Blain-Moraes S, Schaff R, Gruis K, Huggins J, Wren P. Barriers to and Mediators of BrainComputer Interface User Acceptance: Focus Group Findings. Ergonomics. 2012; 55:516-525. [PubMed: 22455595]

[6]. Boly M, Faymonville ME, Peigneux P, Lambermont B, Damas P, Del Fiore G, et al. Auditory processing in severely brain injured patients: Differences between the minimally conscious state and the persistent vegetative state. Arch Neurol. 2004; 61:233-238. [PubMed: 14967772]

[7]. Boly M, Garrido MI, Gosseries O, Bruno M-A, Boveroux P, Schnakers C, et al. Preserved Feedforward But Impaired Top-Down Processes in the Vegetative State. Science. 2011; 332:858-862. [PubMed: 21566197]

[8]. Canu E, Agosta F, Riva N, Sala S, Prelle A, Caputo D, et al. The topography of brain microstructural damage in amyotrophic lateral sclerosis assessed using diffusion tensor MR imaging. AJNR Am J Neuroradiol. 2011; 32:1307-1314. [PubMed: 21680655]

[9]. Chang JL, Lomen-Hoerth C, Murphy J, Henry RG, Kramer JH, Miller BL, et al. A voxel-based morphometry study of patterns of brain atrophy in ALS and ALS/FTLD. Neurology. 2005; 65:75-80. [PubMed: 16009889]

[10]. Cirillo M, Esposito F, Tedeschi G, Caiazzo G, Sagnelli A, Piccirillo G, et al. Widespread microstructural white matter involvement in amyotrophic lateral sclerosis: a whole-brain DTI study. AJNR Am J Neuroradiol. 2012; 33:1102-1108. [PubMed: 22300932]

[11]. Dehaene, S.; Changeux, J-P. Neural mechanisms for access to consciousness. In: Gazzaniga, M., editor. The Cognitive Neurosciences III. MIT Press; 2004.

[12]. Dehaene S, Changeux J-P, Naccache L, Sackur J, Sergent C. Conscious, preconscious, and subliminal processing: a testable taxonomy. Trends in Cognitive Sciences. 2006; 10:204-211. [PubMed: 16603406]

[13]. Dehaene S, Naccache L. Towards a cognitive neuroscience of consciousness: basic evidence and a workspace framework. Cognition. 2001; 79:1-37. [PubMed: 11164022]

[14]. Douaud G, Filippini N, Knight S, Talbot K, Turner MR. Integration of structural and functional magnetic resonance imaging in amyotrophic lateral sclerosis. Brain. 2011; 134:3470-3479. [PubMed: 22075069]

[15]. Farwell LA, Donchin E. Talking off the top of your head: toward a mental prosthesis utilizing event-related brain potentials. Electroencephalography and Clinical Neurophysiology. 1988; 70:510-523. [PubMed: 2461285]

[16]. Filippini N, Douaud G, Mackay CE, Knight S, Talbot K, Turner MR. Corpus callosum involvement is a consistent feature of amyotrophic lateral sclerosis. Neurology. 2010; 75:16451652. [PubMed: 21041787]

[17]. Friston KJ. Functional and effective connectivity: A review. Brain Connectivity. 2011; 1:13-36. [PubMed: 22432952]

[18]. Gourevitch B, Eggermont JJ. Evaluating information transfer between auditory cortical neurons. Journal of Neurophysiology. 2007; 97:2533-2543. [PubMed: 17202243]

[19]. Grosskreutz J, Kaufmann J, Frädrich J, Dengler R, Heinze H-J, Peschel T. Widespread sensorimotor and frontal cortical atrophy in Amyotrophic Lateral Sclerosis. BMC Neurol. 2006; 6:17. [PubMed: 16638121]

[20]. Inuggi A, Riva N, González-Rosa JJ, Amadio S, Amato N, Fazio R, et al. Compensatory movement-related recruitment in amyotrophic lateral sclerosis patients with dominant upper motor neuron signs: An EEG source analysis study. Brain Research. 2011; 1425:37-46. [PubMed: 22030407]

[21]. Kew JJM, Leigh PN, Playford ED, Passingham RE, Goldstein LH, Frackowiak RSJ, et al. Cortical Function in Amyotrophic Lateral Sclerosis A Positron Emission Tomography Study. Brain. 1993; 116:655-680. [PubMed: 8513396]

[22]. Konrad C, Henningsen H, Bremer J, Mock B, Deppe M, Buchinger C, et al. Pattern of cortical reorganization in amyotrophic lateral sclerosis: a functional magnetic resonance imaging study. Experimental Brain Research. 2002; 143:51-56.

[23]. Ku S-W, Lee U, Noh G-J, Jun I-G, Mashour GA. Preferential inhibition of frontal-to-parietal feedback connectivity is a neurophysiologic correlate of general anesthesia in surgical patients. PLoS ONE. 2011; 6:e25155. [PubMed: 21998638] 
[24]. Marincola FM. The trouble with translational medicine. Journal of Internal Medicine. 2011; 270:123-127. [PubMed: 21575085]

[25]. Mitchell J, Borasio G. Amyotrophic lateral sclerosis. The Lancet. 2007; 369:2031-2041.

[26]. Mohammadi B, Kollewe K, Samii A, Krampfl K, Dengler R, Münte TF. Changes of resting state brain networks in amyotrophic lateral sclerosis. Experimental Neurology. 2009; 217:147-153. [PubMed: 19416664]

[27]. Murphy J, Henry R, Lomen-Hoerth C. Establishing subtypes of the continuum of frontal lobe impairment in amyotrophic lateral sclerosis. Arch. Neurol. 2007; 64:330-334. [PubMed: 17353375]

[28]. Naghavi HR, Nyberg L. Common fronto-parietal activity in attention, memory, and consciousness: shared demands on integration? Conscious Cogn. 2005; 14:390-425. [PubMed: 15950889]

[29]. Pascual-Leone A, Walsh V. Fast Backprojections from the Motion to the Primary Visual Area Necessary for Visual Awareness. Science. 2001; 292:510-512. [PubMed: 11313497]

[30]. Rees G, Kreiman G, Koch C. Neural correlates of consciousness in humans. Nature Reviews Neuroscience. 2002; 3:261-270.

[31]. Rose S, Pannek K, Bell C, Baumann F, Hutchinson N, Coulthard A, et al. Direct evidence of intra- and interhemispheric corticomotor network degeneration in amyotrophic lateral sclerosis: an automated MRI structural connectivity study. Neuroimage. 2012; 59:2661-2669. [PubMed: 21893207]

[32]. Sage CA, Van Hecke W, Peeters R, Sijbers J, Robberecht W, Parizel P, et al. Quantitative diffusion tensor imaging in amyotrophic lateral sclerosis: revisited. Hum Brain Mapp. 2009; 30:3657-3675. [PubMed: 19404990]

[33]. Schoenfeld MA, Tempelmann C, Gaul C, Kühnel GR, Düzel E, Hopf J-M, et al. Functional motor compensation in amyotrophic lateral sclerosis. Journal of Neurology. 2005; 252:944-952. [PubMed: 15750701]

[34]. Schreiber T. Measuring Information Transfer. Phys. Rev. Lett. 2000; 85:461-464. [PubMed: 10991308]

[35]. Shao Z, Burkhalter A. Different balance of excitation and inhibition in forward and feedback circuits of rat visual cortex. J. Neurosci. 1996; 16:7353-7365. [PubMed: 8929442]

[36]. Staniek M, Lehnertz K. Symbolic transfer entropy. Phys. Rev. Lett. 2008; 100:158101. [PubMed: 18518155]

[37]. Staniek M, Lehnertz K. Symbolic transfer entropy: inferring directionality in biosignals. Biomed Tech (Berl). 2009; 54:323-328. [PubMed: 19938889]

[38]. Tedeschi G, Trojsi F, Tessitore A, Corbo D, Sagnelli A, Paccone A, et al. Interaction between aging and neurodegeneration in amyotrophic lateral sclerosis. Neurobiol Aging. 2012; 33:886898. [PubMed: 20739098]

[39]. Teismann IK, Warnecke T, Suntrup S, Steinsträter O, Kronenberg L, Ringelstein EB, et al. Cortical processing of swallowing in ALS patients with progressive dysphagia - A magnetoencephalographic study. PLoS ONE. 2011; 6:e19987. [PubMed: 21625445]

[40]. Verstraete E, Veldink JH, Mandl RCW, van den Berg LH, van den Heuvel MP. Impaired Structural Motor Connectome in Amyotrophic Lateral Sclerosis. PLoS ONE. 2011; 6:e24239. [PubMed: 21912680]

[41]. Voss U, Holzmann R, Tuin I, Hobson JA. Lucid dreaming: a state of consciousness with features of both waking and non-lucid dreaming. Sleep. 2009; 32:1191-1200. [PubMed: 19750924]

[42]. Vucic S, Nicholson GA, Kiernan MC. Cortical hyperexcitability may precede the onset of familial amyotrophic lateral sclerosis. Brain. 2008; 131:1540-1550. [PubMed: 18469020]

[43]. Yokota T, Yoshino A, Inaba A, Saito Y. Double cortical stimulation in amyotrophic lateral sclerosis. J Neurol Neurosurg Psychiatry. 1996; 61:596-600. [PubMed: 8971106] 
Highlights for "Altered Cortical Communication in Amyotrophic Lateral Sclerosis"

- We compare cortical communication between individuals with and without ALS.

- We measure directed functional connectivity between frontal and parietal regions.

- Feedback connectivity is not significantly different between groups.

- Feedforward connectivity is significantly higher in individuals with ALS. 
a)

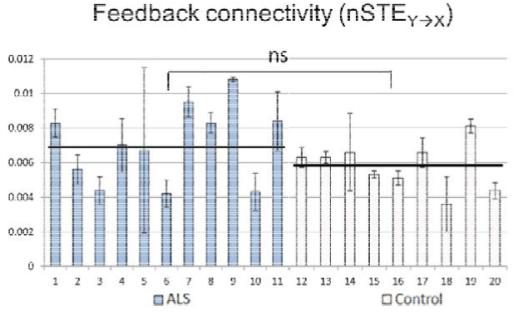

Feedforward connectivity $\left(n S \mathrm{SE}_{X \rightarrow Y}\right)$

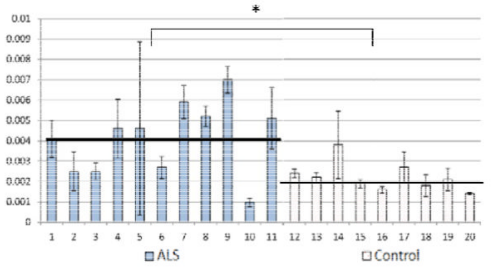

Asymmetry (dimensionless)

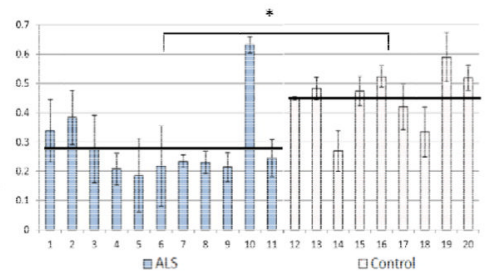

b)

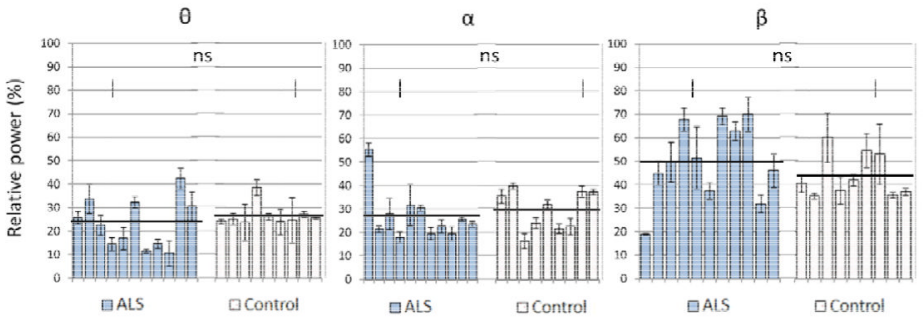

Figure 1.

a) Directed functional connectivity measures between individuals with ALS and agematched controls. Average and standard deviation across three sessions are presented for each participant. b) Relative power spectral densities in frequency bands $\theta: 4-8 \mathrm{~Hz} ; \mathrm{a}: 8-13$ $\mathrm{Hz} ; \beta: 13-30 \mathrm{~Hz}$ for each participant. No significant differences are observed between individuals ALS and age-matched controls. 


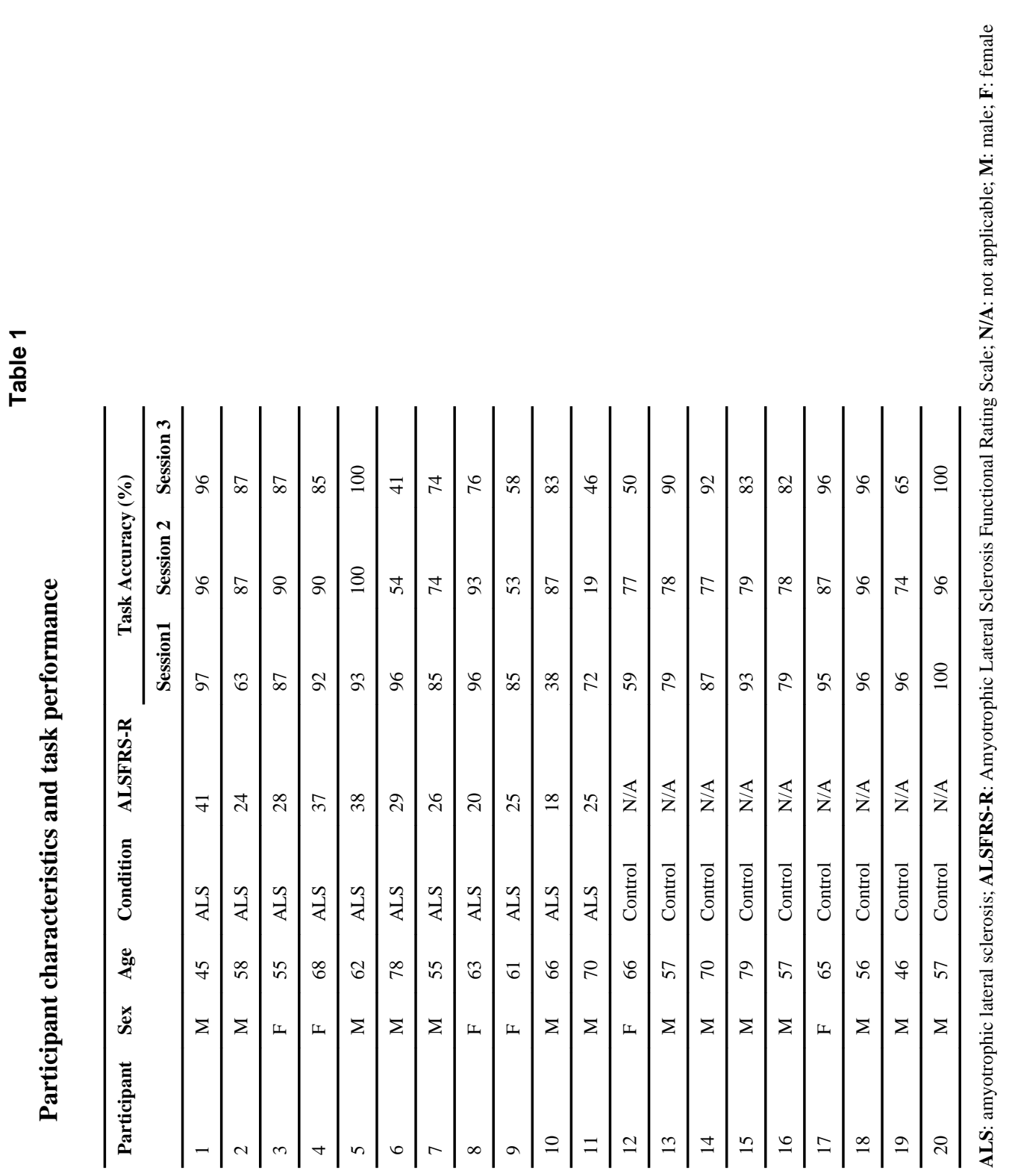

